\title{
Conformation-regulated mechanosensory control via titin domains in cardiac muscle
}

\author{
Tobias Voelkel • Wolfgang A. Linke
}

Received: 5 February 2011 /Revised: 8 February 2011 / Accepted: 9 February 2011 /Published online: 25 February 2011

(C) The Author(s) 2011. This article is published with open access at Springerlink.com

\begin{abstract}
The giant filamentous protein titin is ideally positioned in the muscle sarcomere to sense mechanical stimuli and transform them into biochemical signals, such as those triggering cardiac hypertrophy. In this review, we ponder the evidence for signaling hotspots along the titin filament involved in mechanosensory control mechanisms. On the way, we distinguish between stress and strain as triggers of mechanical signaling events at the cardiac sarcomere. Whereas the Z-disk and M-band regions of titin may be prominently involved in sensing mechanical stress, signaling hotspots within the elastic I-band titin segment may respond primarily to mechanical strain. Common to both stress and strain sensor elements is their regulation by conformational changes in protein domains.
\end{abstract}

Keywords Passive tension · Passive stiffness .

Cardiomyocyte $\cdot$ Cardiac hypertrophy $\cdot$ Signal transduction . Cardiac muscle mechanics

\section{Introduction}

Mechanosensing is likely to be a fundamental property of many living cells. The need to handle mechanical signals is particularly obvious in muscle cells, including cardiomyocytes, which are constantly exposed to external forces and generate forces themselves. The main constituents of a striated muscle cell are the sarcomeres, the contractile units (Fig. 1). A sarcomere has multiple links to cellular

T. Voelkel $\cdot$ W. A. Linke $(\bowtie)$

Department of Cardiovascular Physiology, Institute of Physiology,

Ruhr University Bochum,

MA 3/56,

44780 Bochum, Germany

e-mail: wolfgang.linke@rub.de organelles, the plasma membrane, and the non-sarcomeric cytoskeleton, and it sends out/receives various signals to/ from the sarcoplasm. Such high connectivity makes it a challenge to identify individual components in the whole machinery of mechanotransduction which are perhaps more important than others in sensing mechanical signals. However, recent work has provided evidence for mechanosensory "hotspots" within the sarcomere, located in the Z-disk, I-band, and M-band regions. All these regions are linked by a filamentous network consisting of the giant molecule titin (also known as connectin), which is anchored in the Z-disk, extends as an elastic spring throughout the Iband, and then reaches all the way to the M-band, bound to the thick filament (Fig. 1). Given titin's layout in the sarcomere, the protein seems ideally positioned to act as a sensor of mechanical signals. Indeed, emerging evidence, reviewed and critically discussed below with a focus on cardiomyocytes, suggests that nodal points within the titin filament respond to mechanical stress or strain with conformational changes in protein domains. These changes trigger signaling events eventually leading to altered expression of genes, such as those promoting cardiac hypertrophy.

\section{Stress and strain as mechanical signals acting on titin}

As mechanochemical signaling has become a topic of wide interest in muscle physiology, cell biology, and biophysics, we feel there is a need for a word of caution about the use of the terms "stress" and "strain". These parameters are not always used according to their original meaning in solid mechanics; in fact, it is not uncommon in work on mechanosensing that no distinction is made between mechanical "stress" and "strain". However, 


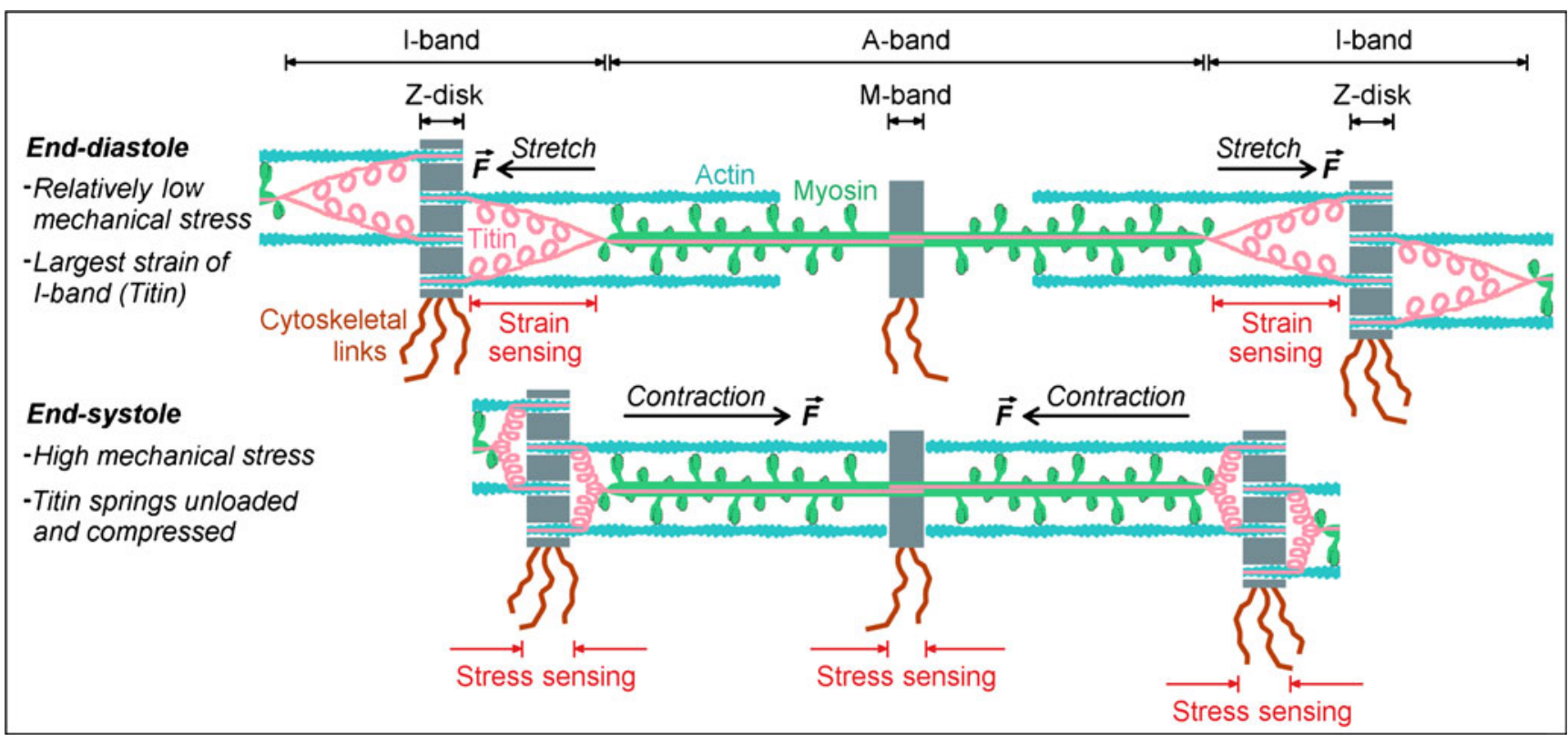

Fig. 1 Schematic of cardiac sarcomeres connected in series, at two distinct states: end diastole (top) and end systole (bottom). For clarity, only the three main filament systems of the sarcomere, actin, myosin,

mechanical stress is force per cross-sectional area (unitPascal or $\mathrm{N} / \mathrm{m}^{2}$ ) and strain is a normalized measure of deformation definable as change in length related to initial length (dimensionless). With strain described as deformation resulting from stress, it is obvious that-regardless of the relationship between these parameters, as in a stressstrain (passive length-tension) curve of a muscle fibersynonymous use is illegitimate.

It does indeed matter to make this distinction in muscle mechanosensing, considering that a large increase in contractile force (or stress) can occur without a substantial change in sarcomere length (or strain), for example in an isometric contraction. In this case, the stress is generated by the sarcomere itself. Or consider a cardiomyocyte being stretched during diastolic distension of the left ventricle: while "passive" stress is relatively low, the elastic titin springs lengthen substantially (Fig. 1). In this example, large sarcomeric I-band strain arises from (low) stress originating from sources outside the myocyte. In the sarcomere, one may intuitively classify the Z-disk and Mband structures, which are barely extensible but exposed to high mechanical stresses during contraction, as stress (force or tension) sensors (Fig. 1). Likewise, titin's $\mathrm{NH}_{2}$-terminal and $\mathrm{COOH}$-terminal regions are firmly anchored at the Zdisk and M-band, respectively, and may be stretched only little, if at all, during normal contraction-relaxation cycles, suggesting these titin segments may be able to sense stress rather than strain. In contrast, the I-band titin springs, readily stretched in diastole and compressed during systolic contraction, may act predominantly as a strain (length or and titin filaments, are shown. Highlighted are the regions in the sarcomere proposed to be involved in stress or strain sensing

stretch) sensor (Fig. 1). Along this line of reasoning, we reserve the term "stress sensor" for Z-disk and M-band titin regions and "strain sensor" for mechanosensory active domains in I-band titin.

Having said this, we will point out later in this review that a stress-sensor or a strain-sensor element responds to a mechanical stimulus ultimately in a similar way: with conformational change(s) in protein domains. In this respect, the distinction between stress and strain sensing is difficult to keep up at the atomic level. A more universal description may be "conformation-regulated mechanosensory control".

\section{Titin gene, protein size, and isoform composition in myocardium}

The specialized mechanosensory functions of titin discussed below are based on a unique protein structure. Mammals have a single titin gene from which many (potentially millions of [25]) isoforms are generated by alternative splicing [40]. The human titin gene has a size of $294 \mathrm{~kb}$ (363 exons) and codes for a maximum of 38,138 aminoacid residues or a 4.2-MDa protein [3]. The largest cardiac titin isoforms are found in fetal hearts, reaching a molecular mass of 3.7 MDa [34, 42, 74, 92]. These isoforms are of the "N2BA" type (Fig. 2). During pre- or perinatal development, the large fetal titin is replaced by smaller size isoforms. In the adult hearts of many mammalian species, the prevalent titin is a 3.0-MDa "N2B" isoform. The normal 


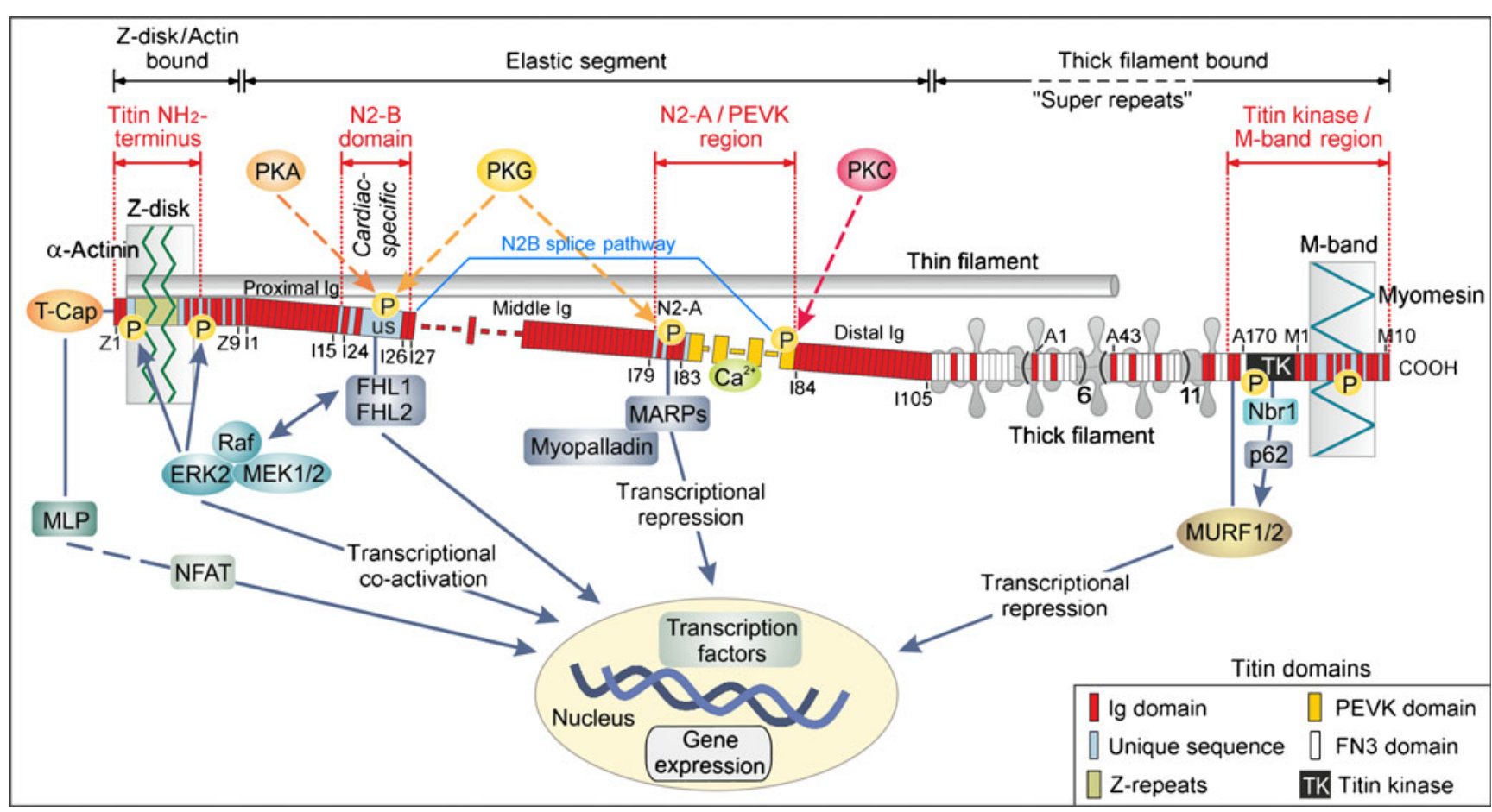

Fig. 2 Titin domain arrangement in the cardiac sarcomere and connectivity within a mechanical signaling network. Shown is the domain structure of the N2BA-titin isoform, but the splice pathway of the N2B isoform is indicated as well (blue line and text). Highlighted are the regions within titin suggested to be involved in mechanosensing: the $\mathrm{NH}_{2}$ terminus in the Z-disk, the N2-B and N2-A/PEVK regions in the I-band, and the $\mathrm{COOH}$ terminus in the M-band. Thick dashed lines indicate differential splicing. Small numbers indicate titin domain numbering according to Ref. [3]. Abbreviations: ERK2 extracellular signal-regulated kinase-2, FHL1/FHL2 four-and-a-half-

adult human left ventricle expresses the titin isoforms at a ratio of $\sim 35 \% \mathrm{~N} 2 \mathrm{BA}$ to $\sim 65 \% \mathrm{~N} 2 \mathrm{~B}[70]$. In a recent mutant rat model, an unusually large, $\sim 3.9 \mathrm{MDa}$, cardiac N2BA titin has been identified, which stays the predominant isoform even in the adult heart [23]. For comparison, adult wild-type rat hearts express $<10 \%$ N2BA and $>90 \%$ N2B isoform [71]. The mutant rats live long with relatively minor heart problems [23], suggesting that a low proportion of N2B titin is not detrimental to rat cardiac function. However, this model could be useful for exploring the role of titin in mechanosensing mechanisms.

The $\mathrm{NH}_{2}$ terminus of the titin molecule is anchored in the Z-disk (Fig. 2) [40]. The titin Z-disk portion is defined as a stretch of nine immunoglobulin-like (Ig) domains (Z1-Z9) interrupted by unique intervening sequences. (Please note that the titin domain numbering used in this review is based upon the nomenclature of Ref. [3].) In Zdisk titin, the Ig domains are constitutively expressed, but repeating sequence motifs, the "Z-repeats", are differentially spliced in different muscle types $[16,86]$. Up to seven of these modules can be expressed, each containing 45 residues.
LIM-domain protein-1 and -2, FN3 domain fibronectin-type-3-like domain of titin, Ig domain immunoglobulin-like domain of titin, $M A R P S$ muscle ankyrin repeat proteins, MEK1/2 MAPK/ERK kinase1 and -2, $M L P$ muscle LIM protein, $M U R F 1 / 2$ muscle-specific RINGfinger proteins- 1 and $-2, N F A T$ nuclear factor of activated $\mathrm{T}$ cells, $N b r 1$ neighbor of BRCA1 gene-1, $P$ titin phosphorylation site, $P K A$ cAMP-dependent protein kinase-A, $P K C \mathrm{Ca}^{2+}$-dependent protein kinase-C $\alpha, P K G$ cGMP-dependent protein kinase-G, T-Cap titin-cap (telethonin), us unique sequence of cardiac N2-B region

The functionally elastic titin segment, the "I-band titin", is thought to begin $\mathrm{COOH}$-terminal to the $\mathrm{Z9}$ domain (Fig. 2). Ig-domain I1 is approximately $100 \mathrm{~nm}$ away from the Z-disk center. The first 15 I-band Ig domains (I1-I15) make up the "proximal" (to the Z-disk) Ig-domain segment, to be distinguished from a series of 22 Ig domains (I84I105) at the other end of the titin-spring region, the "distal" Ig segment. Both these segments are constitutively expressed in the cardiac and skeletal titin isoforms [40]. Another constitutively expressed I-band titin region is an $\sim 180$-amino-acid-long unique sequence just $\mathrm{NH}_{2}$-terminal to the distal Ig segment, representing the $\mathrm{COOH}$-terminal part of the PEVK domain (after the single letter code for proline, glutamic acid, valine, and lysine, the predominant constituting amino acids). Further, only one cardiac-specific segment exists within the whole titin molecule, the "N2-B" domain in the central I-band portion (Fig. 2). The human N2-B domain, which is expressed in both the N2B and N2BA isoforms, comprises three Ig domains (I24/I25; I26) interspersed with a 572-amino-acid-long unique sequence (N2-Bus). The proximal and distal Ig domains, the N2-B domain, and the $\mathrm{COOH}$-terminal PEVK domain make up 
the elastic I-band segment of the cardiac N2B-titin isoform (Fig. 2; see blue line labeled "N2B splice pathway").

Cardiac N2BA-titin isoforms contain, besides all I-band domains of the N2B isoform, two additional I-band segments, which are differentially spliced [12] depending on species, developmental stage, disease state, and location in the heart [56]: a variable-length middle Ig-domain region (I27-I79) and the remainder of the PEVK domain, encoded by more than 100 exons (Fig. 2). In the PEVK domain, up to 60 repeating motifs have been identified, each averaging 28 amino acids encoded by a single exon [22]. Additionally, the central I-band segment of the N2BA titins, but not the N2B isoform, also includes the "N2-A" domain, which comprises four Ig domains (I80-I83) and a few intervening sequences.

A-band titin, the largest part of the molecule, again is constitutively expressed in heart and skeletal muscles. Once titin joins the thick filament and is bound to myosin, a second globular domain type next to the Ig domain appears, the fibronectin-type-3 (FN3) domain, which makes up the majority of A-band titin. Most Ig and FN3 domains in the A-band are arranged in seven-domain and 11-domain super-repeats, repeated six and 11 times, respectively (Fig. 2) [40]. Importantly, titin also belongs to the CaMKinase serine/threonine protein kinase family as it contains a constitutively expressed titin-kinase (TK) domain near the M-band. Almost the entire M-band titin segment (Ig domains M1-M10, interrupted by various unique sequences) is constitutively expressed, but a developmentally regulated splice event affects a unique insertion at the $\mathrm{COOH}$ terminus, Mis-7 [33]. In summary, the majority of titin domains are constitutively expressed in heart and skeletal muscles, whereas differential splicing of exons coding for the elastic I-band region brings about the huge size diversity of titin.

\section{Diversity in titin function}

Titin has long been known to have major mechanical and scaffolding roles in the myocyte. The highly ordered paracrystalline structure of the sarcomere, particularly of the Aband region, would probably be impossible without a "molecular ruler" function of titin, which is now accepted by many but still requires more definite evidence. Titin is also thought to decisively aid in myofibril assembly [10, 90]. An emerging function of titin is that of an integrator of myocyte signaling pathways [37], which will be discussed in more detail below.

The largest body of evidence has been assembled in support of titin's role as a molecular spring. Immunoelectron and immunofluorescence microscopic studies have shown that the I-band region of titin is extensible [14] and contains distinct mechanically active elements, which respond differently to a stretch force: the Ig segments, the PEVK domain, and (in cardiac muscle) the N2-Bus [54, 55, $58,89]$. Single-molecule mechanical studies on native titin or recombinant titin fragments $[31,82,91]$ have established that these I-band titin elements behave like entropic springs with different bending rigidities (or persistence lengths, in terms of polymer elasticity theory; reviewed in Ref. [52]). Accordingly, the three elements extend sequentially during sarcomere stretch [51]: first the Ig segments, which straighten out interdomain linkers (this requires rather low forces), then the PEVK domain, and shortly after also the N2-Bus (which requires the highest stretch forces to be unraveled). Ig-domain unfolding is thought to occur only very infrequently $[49,66]$. It is now established that the stress-strain curve of the three-element I-band-titin spring is the principal determinant of the passive length-tension curve of a cardiomyocyte $[48,56]$.

Additional mechanical functions have been suggested for titin. Optimum active force production depends on titin, as it helps position the myosin filaments in the center of the sarcomere [28]. Further, the (visco)elasticity of the titin springs brings the sarcomere back to slack length following stretch or contraction [77]. Moreover, elastic recoil of the cardiac titin springs acts in support of cardiomyocyte active shortening, whereas viscous drag forces partly arising from interactions between the PEVK domain and the thin filament [38] oppose the shortening [73]. Titin-actin interactions have also been proposed to play some (yet obscure) role in regulating muscle force, which may help stabilize and protect the sarcomere [47]. Finally, titin may be a factor in the length-dependent activation of cardiac muscle [7, 87], a special type of mechanosensing underlying the Frank-Starling law of the heart, which describes an increase in cardiac work output with increased diastolic filling [9]. In conclusion, the titin filaments provide the sarcomere with multiple important mechanical functions. The extensibility of the titin springs is a prerequisite for the sarcomeric I-band region to act as a strain sensor.

\section{Titin connectivity in the myocyte}

A mechanosensory function of titin also requires the association with other structural and signaling molecules. More than 20 interaction partners have been identified for titin, many of which integrate the protein in mechanochemical signal transduction pathways. Interactions occur with all structural motif types found along titin: Ig and FN3 domains, the PEVK segment, and other unique sequences, including the Z-repeats, the N2-Bus, and the titin-kinase domain. The connectivity in the myocyte provided by titin has recently been addressed in detail $[10,48,50]$, and two 
main pathways important for myocyte hypertrophy and protein quality control have been highlighted [37]. A main goal of this review is to ponder the evidence for mechanosensory control mechanisms involving distinct signaling hotspots within titin (Fig. 2): the $\mathrm{NH}_{2}$-terminal segment in the Z-disc, the N2-B and N2-A/PEVK regions in the elastic I-band portion, and the COOH-terminal segment within and adjacent to the M-band.

\section{Role of titin in Z-disk stress sensing}

External mechanical signals (e.g., cardiac hemodynamic load) are transmitted via the extracellular matrix (ECM) and transmembrane protein complexes to the cytoskeletal filament network, which has various links to the Z-disk $[11,29,50,80]$. Conversely, the sarcomeres themselves generate forces, which are transmitted via the Z-disk and the cytoskeletal links to the ECM. Thus, the Z-disk is centrally positioned in a bidirectional force transmission pathway. Importantly, the Z-disk structure experiences both active and passive stresses, as it anchors the actin filaments, on which the myosin motors pull during systolic contraction, as well as the titin springs, which develop elastic forces during diastolic distension. We argue that several regions within Z-disk titin have the potential to participate in mechanical stress sensing. As this review's focus is on titin, we do not intend to exhaustively discuss evidence for the involvement of other Z-disk bound proteins in mechanosensing.

\section{Extreme $\mathrm{NH}_{2}$ terminus of titin}

The two $\mathrm{NH}_{2}$-terminal titin Ig domains, $\mathrm{Z1} / \mathrm{Z} 2$, bind in an exceptionally strong complex to telethonin (also known as T-Cap; Fig. 2). X-ray crystallography demonstrated that telethonin assembles the Z1/Z2 domains of two titin filaments entering the Z-disk from the same half-sarcomere into an anti-parallel sandwich structure [98]. This complex is highly resistant to stretching forces, as multiple hydrogen bonds cross-link beta-strands from either protein [46]. The Z1/Z2-telethonin complex has been suggested to be critical for the Z-disk structure and for anchoring titin in the Z-disk. However, a normal Z-disk appearance observed in skeletal muscles of telethonin knockout mice does not provide support for this idea [62]. Whether Z-disks deficient in telethonin yield under mechanical stress remains to be seen. Telethonin also binds various other proteins, including muscle LIM protein (MLP), which has been implicated in stress sensing [32]. MLP is detected not only at the Z-disk but also in the I-band, at the costameres, in the cytosol, and in the nucleus. Shuttling of MLP to the nucleus activates transcriptional regulators (Fig. 2) and may be pro- hypertrophic [5]. Further, MLP binds to calcineurin, a protein phosphatase triggering translocation of nuclear factor of activated T-cells to the nucleus, where it induces a hypertrophic gene program. Thus, MLP is a signaling molecule in cardiomyocytes. However, the earlier view of the MLP-Z1/Z2-titin-telethonin complex as a stress-sensor element in the Z-disk [32] is unlikely to hold unless it can be shown that mechanical stress actually reaches this complex in the sarcomeric Z-disk. Possibly, the stress is dissipated in other Z-disk regions that do not directly connect to MLP.

\section{Titin Z-repeats}

A Z-disk zone that does respond to mechanical stress with structural changes is where the $\alpha$-actinin molecules crosslink the actin filaments of opposite polarity [59]. Alphaactinin also binds to the Z-repeats of titin (Fig. 2), which themselves associate with actin, so that multiple layers (up to six) of titin- $\alpha$-actinin-actin links are formed [86, 97]. This zone appears in cross-section either like a "smallsquare" lattice formed by actin/titin and the interconnecting $\alpha$-actinin links, or like a "basketweave" lattice with less curved $\alpha$-actinin links [19]. In resting or passively stretched skeletal muscle, the sarcomeres have Z-disks with a smallsquare lattice, but during tetanic contraction or in rigor state the lateral actin-filament spacing is increased by $20 \%$ and the Z-disks adopt the basketweave lattice [19]. Thus, the Zdisk lattice undergoes conformational changes in response to mechanical stress.

The Z-disk lattice was not studied in activated cardiac muscle, but in unstimulated myocardium both the smallsquare and the basketweave lattice forms coexisted, though the latter was much more abundant [20]. The unstimulated cardiac Z-disk thus resembled the Z-disk of tetanized skeletal muscle. However, a remarkable effect was observed when the myocardial samples were treated with $\mathrm{Ca}^{2+}$ chelator EGTA: now the lateral actin-filament spacing decreased by $20 \%$ to a value similar to that of nonactivated skeletal muscle and the cardiac Z-disks exhibited the smallsquare lattice form [18]. These findings were explained by relatively high stresses acting on the Z-disk even in unstimulated myocardium, owing to a high passivetension level and a role for $\mathrm{Ca}^{2+}$ in diastolic tension generation. An alternative possibility is that $\mathrm{Ca}^{2+}$ directly affects the Z-disk lattice independent of mechanical stress. Interestingly, in unstimulated rat myocardium, $90 \%$ of the Z-disk lattice adopted the basketweave form, but in unstimulated dog myocardium only $\sim 70 \%$ [19]. Today we know that passive tension is higher in rat than in dog cardiomyocytes because the rat heart expresses almost exclusively the stiff N2B isoform of titin [71], whereas the dog heart contains the N2B isoform and more compliant N2BA isoforms at similar levels [30]. Therefore, the titin- 
isoform pattern and, consequently, the titin-based passive tension level, could be a factor in determining the Z-disk lattice form. In any case, the presence of two lattice states regulated by mechanical stress suggests a dynamic behavior of the cardiac Z-disk.

In a speculative scenario, mechanical stress might strain the titin Z-repeats along with the $\alpha$-actinin links since the two proteins interact. Alpha-actinin is known to associate with a plethora of structural and signaling molecules, some of which [e.g., calcineurin, MLP, calsarcin, or extracellular signal-regulated kinase (ERK)] have been implicated in cardiac hypertrophic signaling [11, 29]. One can thus envision that a deformation of the Z-disk lattice by mechanical stress causes conformational changes in $\alpha$ actinin and the titin Z-repeats, which could induce hypertrophic signaling pathways. The Z-repeats of titin in complex with actin and $\alpha$-actinin, which are embedded in a signaling network, are a promising candidate for a stresssensor element in the Z-disk.

Actin-binding region of titin at the Z-disk/I-band junction

The titin segment $\mathrm{COOH}$-terminal to the Z-repeats, up to the ninth Ig domain (Z9), reaches beyond the electrondense part of the Z-disk structure up to the so-called N1 line of the sarcomere. This titin segment is barely extensible in normal cardiac sarcomeres, but it extends in response to stretch forces if sarcomeric actin is extracted by gelsolin treatment, suggesting an association with the thin filament $[53,88]$. Binding of the Z9/I1 Ig domains to actin has been confirmed in vitro [53]. Thus, a first anchorage point for the titin filament when it enters the Z-disk region is at the periphery of the Z-disk. This Z-disk titin segment can in theory experience both passive and active forces (as it is actin filament bound) and some of the stress transmitted to the Z-disk might be dissipated at the I-band/Z-disk junction by structural rearrangements in titin domains. Although this sarcomeric region has not yet been explored in terms of a mechanosensor function, one can speculate that stressinduced conformational changes in titin domains at the peripheral Z-disk are able to trigger mechanosensitive signaling events.

A role for Z-disk titin phosphorylation?

Sequence insertions Zis-1 and Zis-5 within Z-disk titin contain consensus sequences for proline-directed kinases. These XSPXR motif repeats are phosphorylated in vitro, for instance by ERK2 (Fig. 2) [16, 83], and this posttranslational modification was proposed to be important during myofibrillogenesis [83]. Several of the phosphosites in Zdisk titin were recently confirmed in a mass-spectrometry study on human skeletal muscle [27]. Since phosphoryla- tion can alter protein-protein interactions, an interesting possibility is that phosphorylation of Z-disk titin residues affects titin linkage to a putative mechanosensory protein network. In this context, we note that not only the titin Zrepeats bind to $\alpha$-actinin but also Zis-1 [41] and Zis-2 [97]. Further, Zis-1 associates with an SH3 domain at the C terminus of nebulin/nebulette [94]. Zis-1 also binds $\gamma$ filamin [41], which has multiple connections in the myocyte stress-response pathway, e.g., to integrin and sarcoglycan at the costameres. External forces transmitted via the costameres can initiate a signaling cascade involving stress-responsive proteins, such as vinculin, melusin, talin, focal-adhesion kinase, integrin-linked kinase, and others, eventually promoting gene expression and cardiomyocyte growth [50]. Thus, phosphorylation of Zis-1 or other Z-disk titin segments could readily alter one or more of these signaling events, with consequences for myocyte stress sensing.

In conclusion, many findings point to a participation of Z-disk titin domains in stress-signaling pathways of the cardiomyocyte, but further evidence is needed. Titin seems predestined to perform stress-sensing functions as one of several important components in a highly connected Zdisk-associated protein network.

\section{I-band titin-based strain sensing}

The I-band titin segment has been established as a threeelement molecular spring and principal determinant of cardiomyocyte passive tension [48, 56]. However, an increasing number of ligands found to be associated with the titin springs have prompted new concepts about a putative strain-sensor role for this titin region [50]. We will discuss two segments in I-band titin, the cardiac-specific N2-B domain and the N2-A/PEVK region, which may be focal points of mechanosensing.

\section{Strain sensing via the titin N2-B domain}

The cardiac-specific N2-B titin domain is a hotspot for protein-protein interactions where hypertrophy signaling pathways converge [56]. Especially involved in ligand binding is the large unique sequence insertion (N2-Bus) between Ig domains I25 and I26 (Fig. 2). The N2-Bus is one of the molecular-spring elements in cardiac titin and begins to unravel at passive stress levels reached at high physiological sarcomere lengths [58]. The N2-Bus interacts, among others, with four-and-a-half-LIM-domain proteins, FHL1 and FHL2 [43, 85], which are transcriptional co-activators (Fig. 2). The FHL proteins are involved in a host of protein interactions and they can translocate from the sarcomere and cytosol to the nucleus. FHL2 was shown 
to also target metabolic enzymes to the I-band [43]. FHL1 was suggested to form a mechanosensory complex at the N2-Bus with members of the mitogen activated protein kinase (MAPK) family, including MAPK/ERK kinase-1 and -2 (MEK1/2) and ERK2, as well as their activator Raf1 (Fig. 2) [85]. This complex could act as a bona fide stretch transducer. When the N2-Bus unravels due to a stretch force, activated ERK2 unbinds from the N2-Bus and shuttles to the nucleus where it induces a hypertrophic gene program. If FHL1 is missing from the complex, e.g., in FHL1-deficient mouse hearts, the MAPK complex on N2-B titin is destabilized, myofibrillar compliance is increased, and ERK2 activation is depressed. Indeed, hypertrophy in response to cardiac overload is induced to a lesser degree in FHL1-knockout than in wild-type mouse hearts [85]. Since the Raf-1-MAPK pathway is activated by various extracellular signals, including those mediated by G-protein coupled receptors, strain sensing via the N2-Bus-FHLMAPK complex could represent a general mechanism of hypertrophic gene activation in cardiac muscle.

The N2-Bus is optimally suited to act as a strain sensor. Since it does not measurably extend at the short to intermediate sarcomere-length range [58], its strain-sensor function may not be activated until a high degree of diastolic distension is reached, as occurs, e.g., during volume overload of the left ventricle. Under normal loading conditions, the N2-Bus might not be sufficiently stretched to induce ERK2 shuttling to the nucleus and a significant hypertrophic response. Thus, the N2-Bus-FHL-MAPK complex could be a prominent factor in the development of pathological cardiac hypertrophy.

\section{The N2-A and PEVK titin regions}

A second signaling node in I-band titin is the N2-A region (Fig. 2). Binding partners of this region can be induced by stretch, specifically the three homologous muscle ankyrin repeat proteins (MARPs), cardiac ankyrin repeat protein (CARP), diabetes-related ankyrin repeat protein (DARP), and ankyrin-repeat domain-protein-2, which themselves associate with myopalladin [65]. In neonatal cardiomyocytes, CARP and DARP were upregulated in response to cyclic mechanical strain and were then localized to both the myofibrillar I-bands and the nucleus. Nuclear shuttling of MARPs is thought to promote transcriptional repression and muscle atrophy (Fig. 2). However, mice lacking all three MARP family members appeared phenotypically normal [4]. A different issue is whether titin domains within the N2-A region in fact undergo conformational changes or not in response to physiological force or stretch levels. One can expect that the linker sequences connecting the Ig domains become deformed on sarcomere stretch. The Ig domains of the N2-A domain have a mechanical stability similar to that of other I-band titin domains, and their unfolding probability therefore is low [35]. In light of these findings, the role of the N2-A-MARP connection in mechanosensing needs clarification.

Nearly one third of the exons in the human titin gene code for the alternatively spliced PEVK domain, a segment made up of conserved alternating motifs of 26-28 amino acids (PPAK repeats) separated by regions rich in glutamic acid (polyE motifs) [22]. The three conformational states found in the PEVK domain, polyproline-II helix, beta-turn, and unordered coil [61], could be important for the elastic properties of this domain. Evidence suggests that the PEVK domain behaves mainly as an "entropic spring" and unravels in stretched sarcomeres, which generates relatively high passive-tension levels [49, 54, 55, 89]. The PEVK segment was confirmed to interact with $\mathrm{SH} 3$ domains in nebulin and may bind to $\mathrm{SH} 3$ domains in other proteins as well, implying a role for this segment in sarcomere assembly and perhaps in mechanosensing [60]. Moreover, the stiffness of the PEVK segment is increased by $\mathrm{Ca}^{2+}$ binding [39]. An intriguing link to the thin filament arises from the actin-binding propensity of the PEVK domain [38, $57,69,96]$, which is a source of viscoelastic load in myocardium [38, 73]. A possibility therefore is that stretching the sarcomere and extending the PEVK domain also affects the conformational state of thin-filament protein(s), with consequences for contractile properties. In conclusion, the PEVK segment has a major function as a molecular spring whose mechanical properties are likely to be modified in the sarcomere by $\mathrm{Ca}^{2+}$ and actin binding. Direct evidence for a role of the PEVK domain in mechanosensing is not available, but this issue may be worth following up.

\section{Titin I-band segment knockout models}

Support for a strain-sensor concept involving I-band titin comes from two genetic mouse models, each with a deletion of a different titin spring segment. Deletion of the N2-B region resulted in elevated cardiac titin stiffness and severe diastolic dysfunction [81]. The molecular length of the PEVK segment was higher in N2-B knockout than in wild-type sarcomeres, presumably as a compensation for the lack of N2-Bus extensibility. Notably, FHL2 was downregulated and the hearts were atrophic. This phenotype contrasted with that of a knockout model for the constitutively expressed PEVK segment, which also had diastolic dysfunction but showed cardiac hypertrophy [21]. In this model, cardiac titin stiffness was also increased. The molecular length of the N2-Bus was higher than in wildtype sarcomeres to compensate for the lack of PEVK extensibility, and both FHL1 and FHL2 were upregulated in PEVK-knockout hearts. The hypertrophy was explained by 
activation of the N2-Bus-associated strain sensor because more FHL1 would bind to the overly stretched N2-Bus, and more shuttling of ERK2 to the nucleus could occur. Taken together, it is remarkable that passive tension was increased in both models, whereas hearts with PEVK knockout were hypertrophic, but those with N2-B knockout were atrophic. This suggests that the titin-based passive stress level was not the critical parameter for the hypertrophy/atrophy response. Of more crucial importance seem to be the different stretch states of the molecular spring elements in titin. Hence, the signaling hotspots in I-band titin, and the N2-Bus in particular, may indeed function like a strain sensor rather than a stress sensor.

\section{Posttranslational modifications of I-band titin}

Protein kinases, PKA and cGMP-dependent PKG, both phosphorylate the cardiac-specific N2-Bus in titin (Fig. 2) $[6,13,35,36]$. This phosphorylation, which in human hearts was mapped to serine 469 [35], reduces titin-based myocardial stiffness. In contrast, protein kinase-C $\alpha$ phosphorylates the constitutively expressed PEVK domain (Fig. 2) at two conserved residues, S26 and $\mathrm{S} 170$, in mouse and porcine hearts, and this increases titin-based stiffness [26]. The altered titin stiffness is brought about by an effect of phosphate binding on the bending rigidity of the titin segment [26, 35]. This bending rigidity can be parameterized in entropic elasticity models, such as the "wormlike-chain model", by the persistence length, Lp [52]. Phosphorylation by PKG increases Lp of a recombinantly expressed N2-Bus, as measured by single-molecule atomic force microscopy (AFM) force spectroscopy [35]. In contrast, phosphorylation by $\mathrm{PKC} \alpha$ decreases Lp of a recombinant PEVK domain [26]. Why phosphorylation of some sites in I-band titin decreases Lp, whereas phosphorylation of other sites increases it, remains unclear. In any case, an increase in $\mathrm{Lp}$ will lower the force required to stretch the N2-Bus. Then, at the same force level, the titin segment will be stretched to a longer length than before phosphorylation. The opposite is true for the PEVK segment. Thus, phosphorylation could also modulate the strain-sensor properties of the N2-Bus and the PEVK domain.

Another type of posttranslational modification has been shown to occur in the N2-Bus under oxidizing conditions: the cysteines, of which six are present in the human N2-Bus, can form up to three intramolecular disulfide bonds [24]. This disulfide bonding, which presumably occurs only under some disease conditions, elevates titin-based stiffness of the myocardium. Since the extensibility of the N2-Bus will be reduced, the stretchsensor function of the N2-Bus-FHL-MAPK complex could be impaired as well.
In summary, various lines of evidence implicate distinct Iband titin regions in mechanical strain sensing. In particular, the large unique sequences, the N2-Bus and perhaps the PEVK domain, appear to be part of strain-sensing complexes. Mechanistically, stretch-induced unraveling of these domains could affect the interaction with signaling molecules and induce hypertrophy-promoting pathways.

\section{M-band titin-based stress sensing}

The titin-kinase domain as a mechanosensor

Perhaps the most convincing evidence yet for a role of titin in mechanosensing comes from work on the titin-kinase (TK) domain at the $\mathrm{COOH}$ terminus of titin (Fig. 2), which will be covered in more detail elsewhere in this issue. Phosphorylation of a tyrosine residue in the TK domain plays a role in the complex activation process of this autoinhibited serine/threonine kinase [63]. Although the TK shows some homology to $\mathrm{Ca}^{2+} /$ calmodulin regulated myosin light chain kinases, it is activated by a unique mechanism involving removal of the $\mathrm{COOH}$-terminal autoinhibitory tail by yet unknown protein factor(s) [63] or stretch forces [78]. Single-molecule AFM force spectroscopy demonstrated that exposure of recombinant TK domain to stretch forces causes a conformational change required for ATP binding and access of the autoinhibitory tyrosine $[78,79]$. Confirmation of this mechanism in vivo, which may be a daunting task, would firmly establish the TK domain as a highly specialized mechanosensor important in the stress-induced adaptation of myocyte function.

The TK domain signals via different pathways to the nucleus to induce changes in muscle gene expression (Fig. 2). A zinc-finger protein, neighbor-of-BRCA1-gene-1 (Nbr1), binds to the activated TK domain and forms a signaling complex with p62/SQSTM1. This protein in turn recruits one of the muscle-specific RING finger proteins, MURF2, to the sarcomere [45]. The MURF isoforms can shuttle to the nucleus, where they repress transcription and promote muscle atrophy, likely via inactivation of serum response factor. Located just $\mathrm{NH}_{2}$-terminal to the TK domain are the A-band titin domains, A168-A170, which interact with both MURF1 $[8,64,67]$ and MURF2 [76, 95] to provide another link to nuclear signaling pathways (Fig. 2). A tamoxifen-inducible deletion of the TK region (including the MURF1 binding site) in adult mouse hearts produced severe cardiac hypertrophy and congestive heart failure, associated with an attenuated response to adrenergic stimulation and extracellular $\mathrm{Ca}^{2+}$ [75]. Knockout mouse models for the MURF isoforms confirmed a role for the MURF proteins in muscle atrophy, but also suggested that MURF functions may require synergistic action of the different isoforms [93]. In 
summary, evidence supports the idea that a signalosome complex at the TK domain is activated by mechanical stress via conformational changes in the TK domain. The mechanosensitive pathways induced by activating the TK domain balance hypertrophy versus atrophy promoting signals.

Stability and elasticity provided by the M-band titin region

The $\mathrm{COOH}$ terminus of titin in the M-band is involved in numerous protein-protein interactions $[1,44,56]$. Targeted homozygous deletion of the entire M-band-titin region in cardiomyocytes prevents sarcomere formation [68], demonstrating the importance of this titin segment for thickfilament assembly. The titin Ig domain M4 interacts with myomesin, which in turn anchors M-band titin to the myosin rod [72]. The titin M4 domain is not far from the Mis-4 sequence insertion, which can be phosphorylated by prolinedirected kinases, such as ERK2 (Fig. 2), a modification suggested to be important during muscle development [17]. M-band titin also binds to the giant protein obscurin and its small homologue obscurin-like-1, but these proteins may not have a mechanical function [15]. Thus, the mechanical stability of the M-band is determined by myomesin complexed to M-band titin and myosin [1]. M-band bending and axial misalignment of the thick filaments are observed in contracting muscle, which result mainly from shear stresses acting on the sarcomere [1]. To protect the sarcomere structure during intense and sustained mechanical stress, and to induce the stress-responsive machinery controlling protein synthesis, the myomesin-titin-myosin connection is likely to be of critical importance.

\section{Proposed mechanism of M-band stress sensing}

The M-band is more compliant than the Z-disk, at least in transverse direction [2]. However, if M-band stiffness were too low, a function in sarcomere stability would not be possible [84]. Like the Z-disk, the M-band requires mechanical links to the extra-sarcomeric cytoskeleton to support sarcomere stability and mechanosensing [84]. If the M-band lattice is deformed during contractile activity, owing to the shear forces between adjacent thick filaments [1], mechanical stress acting on the myomesin-titin-myosin links should also be sensed by the titin-kinase domain. The TK will be activated to launch the mechanochemical signaling cascade described above [45, 78]. Importantly, the TK domain is predicted to be in an active conformation not until active forces reach a critical level [84]. In relaxed muscle or at low active tension, when the M-band structure remains stable, TK activity is inhibited. Thus, the M-band stress-detection system is ideally suited for sensing the actual workload on a sarcomere and responding with appropriate adjustments of muscle gene expression.

\section{Conclusions and outlook}

We have critically reviewed current evidence for the involvement of titin regions in mechanosensory signal transduction. Some evidence is more speculative, such as that suggesting a role of Z-disk titin in stress sensing. Stronger evidence has been provided in support of a crucial role of I-band titin segments, especially the cardiac-specific N2-B domain, in strain sensing. Conclusive evidence is also available for the involvement of M-band titin, particularly the titin-kinase domain, in stress sensing. Both the stress-sensor and the strain-sensor elements in titin function by responding to a force or to a stretch with conformational changes, which can alter the interaction with signaling proteins. Unbound transcriptional co-activator/repressor molecules can thus shuttle to the nucleus and promote/depress hypertrophic gene activation. This "conformation-regulated mechanosensory control" via titin domains could be at the heart of many mechanosensing processes in the myocyte.

Many issues still require further study. Obviously, the role of titin in Z-disk stress sensing requires clarification, as does the involvement of some I-band titin segments (such as the N2-A region or the PEVK domain) in stress or strain sensing. An exciting area for future research will be how the proposed mechanisms of titin-based mechanosensing are integrated in the signaling network of the myocyte in vivo. Further, while it is now established that tensile stress or strain can induce mechanical signaling complexes on titin, we know little or nothing about other types of mechanical stress, e.g., compressive forces acting on titin in systole (Fig. 1), and whether or how they are sensed by the titin filament and possibly be transformed into specific signals triggering/suppressing muscle gene activity. Methodologically, a combination of cellular and molecular mechanics approaches, elucidation of titinligand complexes to the atomic detail by X-ray crystallography, and novel live-cell imaging techniques should pave the way for a better understanding of the mechanosensing functions of titin and the sarcomere.

Acknowledgments This work was supported by grants from the German Research Foundation [Li 690/7-2 (KFO 155); Li 690/6-3] and from the European Union (FP7-MEDIA).

Open Access This article is distributed under the terms of the Creative Commons Attribution Noncommercial License which permits any noncommercial use, distribution, and reproduction in any medium, provided the original author(s) and source are credited.

\section{References}

1. Agarkova I, Perriard JC (2005) The M-band: an elastic web that crosslinks thick filaments in the center of the sarcomere. Trends Cell Biol 15:477-485 
2. Akiyama N, Ohnuki Y, Kunioka Y, Saeki Y, Yamada T (2006) Transverse stiffness of myofibrils of skeletal and cardiac muscles studied by atomic force microscopy. J Physiol Sci 56:145-151

3. Bang ML, Centner T, Fornoff F, Geach AJ, Gotthardt M, McNabb M, Witt CC, Labeit D, Gregorio CC, Granzier H, Labeit S (2001) The complete gene sequence of titin, expression of an unusual approximately $700-\mathrm{kDa}$ titin isoform, and its interaction with obscurin identify a novel Z-line to I-band linking system. Circ Res 89:1065-1072

4. Barash IA, Bang ML, Mathew L, Greaser ML, Chen J, Lieber RL (2007) Structural and regulatory roles of muscle ankyrin repeat protein family in skeletal muscle. Am J Physiol Cell Physiol 293:C218-C227

5. Boateng SY, Senyo SE, Qi L, Goldspink PH, Russell B (2009) Myocyte remodeling in response to hypertrophic stimuli requires nucleocytoplasmic shuttling of muscle LIM protein. J Mol Cell Cardiol 47:426-435

6. Borbély A, Falcao-Pires I, van Heerebeek L, Hamdani N, Edes I, Gavina C, Leite-Moreira AF, Bronzwaer JG, Papp Z, van der Velden J, Stienen GJ, Paulus WJ (2009) Hypophosphorylation of the Stiff N2B titin isoform raises cardiomyocyte resting tension in failing human myocardium. Circ Res 104:780-786

7. Cazorla O, Wu Y, Irving TC, Granzier H (2001) Titin-based modulation of calcium sensitivity of active tension in mouse skinned cardiac myocytes. Circ Res 88:1028-1035

8. Centner T, Yano J, Kimura E, McElhinny AS, Pelin K, Witt CC, Bang ML, Trombitas K, Granzier H, Gregorio CC, Sorimachi H, Labeit S (2001) Identification of muscle specific ring finger proteins as potential regulators of the titin kinase domain. $\mathrm{J}$ Mol Biol 306:717-726

9. de Tombe PP, Mateja RD, Tachampa K, Mou YA, Farman GP, Irving TC (2010) Myofilament length dependent activation. J Mol Cell Cardiol 48:851-858

10. Ehler E, Gautel M (2008) The sarcomere and sarcomerogenesis. Adv Exp Med Biol 642:1-14

11. Frank D, Frey N (2011) Cardiac Z-disc signaling network. J Biol Chem (Epub ahead of print Jan. 21, 2011)

12. Freiburg A, Trombitas K, Hell W, Cazorla O, Fougerousse F, Centner T, Kolmerer B, Witt C, Beckmann JS, Gregorio CC, Granzier H, Labeit S (2000) Series of exon-skipping events in the elastic spring region of titin as the structural basis for myofibrillar elastic diversity. Circ Res 86:1114-1121

13. Fukuda N, Wu Y, Nair P, Granzier HL (2005) Phosphorylation of titin modulates passive stiffness of cardiac muscle in a titin isoform-dependent manner. J Gen Physiol 125:257-271

14. Fürst DO, Osborn M, Nave R, Weber K (1988) The organization of titin filaments in the half-sarcomere revealed by monoclonal antibodies in immunoelectron microscopy: a map of ten nonrepetitive epitopes starting at the $\mathrm{Z}$ line extends close to the $\mathrm{M}$ line. J Cell Biol 106:1563-1572

15. Gautel M (2011) The sarcomeric cytoskeleton: who picks up the strain? Curr Opin Cell Biol (Epub ahead of print Dec. 27, 2010)

16. Gautel M, Goulding D, Bullard B, Weber K, Furst DO (1996) The central Z-disk region of titin is assembled from a novel repeat in variable copy numbers. J Cell Sci 109:2747-2754

17. Gautel M, Leonard K, Labeit S (1993) Phosphorylation of KSP motifs in the $\mathrm{C}$-terminal region of titin in differentiating myoblasts. EMBO J 12:3827-3834

18. Goldstein MA, Michael LH, Schroeter JP, Sass RL (1989) Two structural states of Z-bands in cardiac muscle. Am J Physiol 256: H552-H559

19. Goldstein MA, Schroeter JP, Michael LH (1991) Role of the Z band in the mechanical properties of the heart. FASEB J 5:2167-2174

20. Goldstein MA, Schroeter JP, Sass RL (1979) The Z lattice in canine cardiac muscle. J Cell Biol 83:187-204

21. Granzier HL, Radke MH, Peng J, Westermann D, Nelson OL, Rost K, King NM, Yu Q, Tschöpe C, McNabb M, Larson DF,
Labeit S, Gotthardt M (2009) Truncation of titin's elastic PEVK region leads to cardiomyopathy with diastolic dysfunction. Circ Res 105:557-564

22. Greaser M (2001) Identification of new repeating motifs in titin. Proteins 43:145-149

23. Greaser ML, Warren CM, Esbona K, Guo W, Duan Y, Parrish AM, Krzesinski PR, Norman HS, Dunning S, Fitzsimons DP, Moss RL (2008) Mutation that dramatically alters rat titin isoform expression and cardiomyocyte passive tension. J Mol Cell Cardiol 44:983-991

24. Grützner A, Garcia-Manyes S, Kötter S, Badilla CL, Fernandez JM, Linke WA (2009) Modulation of titin-based stiffness by disulfide bonding in the cardiac titin N2-B unique sequence. Biophys J 97:825-834

25. Guo W, Bharmal SJ, Esbona K, Greaser ML (2010) Titin diversityalternative splicing gone wild. J Biomed Biotechnol 2010: 753675

26. Hidalgo C, Hudson B, Bogomolovas J, Zhu Y, Anderson B, Greaser M, Labeit S, Granzier H (2009) PKC phosphorylation of titin's PEVK element: a novel and conserved pathway for modulating myocardial stiffness. Circ Res 105:631-638

27. Højlund K, Bowen BP, Hwang H, Flynn CR, Madireddy L, Geetha T, Langlais P, Meyer C, Mandarino LJ, Yi Z (2009) In vivo phosphoproteome of human skeletal muscle revealed by phosphopeptide enrichment and HPLC-ESI-MS/MS. J Proteome Res 8:4954-4965

28. Horowits R, Maruyama K, Podolsky RJ (1989) Elastic behavior of connectin filaments during thick filament movement in activated skeletal muscle. J Cell Biol 109:2169-2176

29. Hoshijima M (2006) Mechanical stress-strain sensors embedded in cardiac cytoskeleton: $\mathrm{Z}$ disk, titin, and associated structures. Am J Physiol Heart Circ Physiol 290:H1313-H1325

30. Jaber WA, Maniu C, Krysiak J, Shapiro BP, Meyer DM, Linke WA, Redfield MM (2008) Titin isoforms, extracellular matrix, and global chamber remodeling in experimental dilated cardiomyopathy: functional implications and mechanistic insight. Circ Heart Fail 1:192-199

31. Kellermayer MS, Smith SB, Granzier HL, Bustamante C (1997) Folding-unfolding transitions in single titin molecules characterized with laser tweezers. Science 276:1112-1116

32. Knoll R, Hoshijima M, Hoffman HM, Person V, LorenzenSchmidt I, Bang ML, Hayashi T, Shiga N, Yasukawa H, Schaper W, McKenna W, Yokoyama M, Schork NJ, Omens JH, McCulloch AD, Kimura A, Gregorio CC, Poller W, Schaper J, Schultheiss HP, Chien KR (2002) The cardiac mechanical stretch sensor machinery involves a Z-disc complex that is defective in a subset of human dilated cardiomyopathy. Cell 111:943-955

33. Kolmerer B, Olivieri N, Witt CC, Herrmann BG, Labeit S (1996) Genomic organization of $\mathrm{M}$ line titin and its tissuespecific expression in two distinct isoforms. $\mathrm{J}$ Mol Biol 256:556-563

34. Kruger M, Kohl T, Linke WA (2006) Developmental changes in passive stiffness and myofilament $\mathrm{Ca} 2+$ sensitivity due to titin and troponin-I isoform switching are not critically triggered by birth. Am J Physiol Heart Circ Physiol 291:H496-H506

35. Krüger M, Kötter S, Grützner A, Lang P, Andresen C, Redfield MM, Butt E, dos Remedios CG, Linke WA (2009) Protein kinase $\mathrm{G}$ modulates human myocardial passive stiffness by phosphorylation of the titin springs. Circ Res 104:87-94

36. Kruger M, Linke WA (2006) Protein kinase-A phosphorylates titin in human heart muscle and reduces myofibrillar passive tension. J Muscle Res Cell Motil 27:435-444

37. Kruger M, Linke WA (2011) The giant protein titin: a regulatory node that integrates myocyte signaling pathways. J Biol Chem (Epub ahead of print Jan. 21, 2011) 
38. Kulke M, Fujita-Becker S, Rostkova E, Neagoe C, Labeit D, Manstein DJ, Gautel M, Linke WA (2001) Interaction between PEVK-titin and actin filaments: origin of a viscous force component in cardiac myofibrils. Circ Res 89:874-881

39. Labeit D, Watanabe K, Witt C, Fujita H, Wu Y, Lahmers S, Funck T, Labeit S, Granzier H (2003) Calcium-dependent molecular spring elements in the giant protein titin. Proc Natl Acad Sci USA 100:13716-13721

40. Labeit S, Kolmerer B (1995) Titins: giant proteins in charge of muscle ultrastructure and elasticity. Science 270:293-296

41. Labeit S, Lahmers S, Burkart C, Fong C, McNabb M, Witt S, Labeit D, Granzier H (2006) Expression of distinct classes of titin isoforms in striated and smooth muscles by alternative splicing, and their conserved interaction with filamins. J Mol Biol 362:664-681

42. Lahmers S, Wu Y, Call DR, Labeit S, Granzier H (2004) Developmental control of titin isoform expression and passive stiffness in fetal and neonatal myocardium. Circ Res 94:505513

43. Lange S, Auerbach D, McLoughlin P, Perriard E, Schafer BW, Perriard JC, Ehler E (2002) Subcellular targeting of metabolic enzymes to titin in heart muscle may be mediated by DRAL/FHL2. J Cell Sci 115:4925-4936

44. Lange S, Ehler E, Gautel M (2006) From A to Z and back? Multicompartment proteins in the sarcomere. Trends Cell Biol $16: 11-18$

45. Lange S, Xiang F, Yakovenko A, Vihola A, Hackman P, Rostkova E, Kristensen J, Brandmeier B, Franzen G, Hedberg B, Gunnarsson LG, Hughes SM, Marchand S, Sejersen T, Richard I, Edström L, Ehler E, Udd B, Gautel M (2005) The kinase domain of titin controls muscle gene expression and protein turnover. Science 308:1599-1603

46. Lee EH, Gao M, Pinotsis N, Wilmanns M, Schulten K (2006) Mechanical strength of the titin Z1Z2-telethonin complex. Structure 14:497-509

47. Leonard TR, Herzog W (2010) Regulation of muscle force in the absence of actin-myosin-based cross-bridge interaction. Am J Physiol Cell Physiol 299:C14-C20

48. LeWinter MM, Granzier H (2010) Cardiac titin: a multifunctional giant. Circulation 121:2137-2145

49. Li H, Linke WA, Oberhauser AF, Carrion-Vazquez M, Kerkvliet JG, Lu H, Marszalek PE, Fernandez JM (2002) Reverse engineering of the giant muscle protein titin. Nature 418:9981002

50. Linke WA (2008) Sense and stretchability: the role of titin and titin-associated proteins in myocardial stress-sensing and mechanical dysfunction. Cardiovasc Res 77:637-648

51. Linke WA, Fernandez JM (2002) Cardiac titin: molecular basis of elasticity and cellular contribution to elastic and viscous stiffness components in myocardium. J Muscle Res Cell Motil 23:483-497

52. Linke WA, Grützner A (2008) Pulling single molecules of titin by AFM - recent advances and physiological implications. Pflügers Arch 456:101-115

53. Linke WA, Ivemeyer M, Labeit S, Hinssen H, Ruegg JC, Gautel M (1997) Actin-titin interaction in cardiac myofibrils: probing a physiological role. Biophys J 73:905-919

54. Linke WA, Ivemeyer M, Mundel M, Stockmeier MR, Kolmerer B (1998) Nature of PEVK-titin elasticity in skeletal muscle. Proc Natl Acad Sci USA 95:8052-8057

55. Linke WA, Ivemeyer M, Olivieri N, Kolmerer B, Ruegg JC, Labeit S (1996) Towards a molecular understanding of the elasticity of titin. J Mol Biol 261:62-71

56. Linke WA, Krüger M (2010) The giant protein titin as an integrator of myocyte signaling pathways. Physiol Bethesda 25:186-198

57. Linke WA, Kulke M, Li H, Fujita-Becker S, Neagoe C, Manstein DJ, Gautel M, Fernandez JM (2002) PEVK domain of titin: an entropic spring with actin-binding properties. J Struct Biol 137:194-205
58. Linke WA, Rudy DE, Centner T, Gautel M, Witt C, Labeit S, Gregorio CC (1999) I-band titin in cardiac muscle is a threeelement molecular spring and is critical for maintaining thin filament structure. J Cell Biol 146:631-644

59. Luther PK (2009) The vertebrate muscle Z-disc: sarcomere anchor for structure and signalling. J Muscle Res Cell Motil 30:171-185

60. Ma K, Forbes JG, Gutierrez-Cruz G, Wang K (2006) Titin as a giant scaffold for integrating stress and Src homology domain 3mediated signaling pathways: the clustering of novel overlap ligand motifs in the elastic PEVK segment. J Biol Chem 281:27539-27556

61. Ma K, Wang K (2003) Malleable conformation of the elastic PEVK segment of titin: non-co-operative interconversion of polyproline II helix, beta-turn and unordered structures. Biochem J 374:687-695

62. Markert CD, Meaney MP, Voelker KA, Grange RW, Dalley HW, Cann JK, Ahmed M, Bishwokarma B, Walker SJ, Yu SX, Brown M, Lawlor MW, Beggs AH, Childers MK (2010) Functional muscle analysis of the Tcap knockout mouse. Hum Mol Genet 19:2268-2283

63. Mayans O, van der Ven PF, Wilm M, Mues A, Young P, Furst DO, Wilmanns M, Gautel M (1998) Structural basis for activation of the titin kinase domain during myofibrillogenesis. Nature 395:863-869

64. McElhinny AS, Kakinuma K, Sorimachi H, Labeit S, Gregorio CC (2002) Muscle-specific RING finger-1 interacts with titin to regulate sarcomeric $\mathrm{M}$-line and thick filament structure and may have nuclear functions via its interaction with glucocorticoid modulatory element binding protein-1. J Cell Biol 157:125-136

65. Miller MK, Bang ML, Witt CC, Labeit D, Trombitas C, Watanabe K, Granzier H, McElhinny AS, Gregorio CC, Labeit S (2003) The muscle ankyrin repeat proteins: CARP, ankrd2/Arpp and DARP as a family of titin filament-based stress response molecules. J Mol Biol 333:951-964

66. Minajeva A, Kulke M, Fernandez JM, Linke WA (2001) Unfolding of titin domains explains the viscoelastic behavior of skeletal myofibrils. Biophys J 80:1442-1451

67. Mrosek M, Labeit D, Witt S, Heerklotz H, von Castelmur E, Labeit S, Mayans O (2007) Molecular determinants for the recruitment of the ubiquitin-ligase MuRF-1 onto M-line titin. FASEB J 21:1383-1392

68. Musa H, Meek S, Gautel M, Peddie D, Smith AJ, Peckham M (2006) Targeted homozygous deletion of M-band titin in cardiomyocytes prevents sarcomere formation. J Cell Sci 119:4322-4331

69. Nagy A, Cacciafesta P, Grama L, Kengyel A, Malnasi-Csizmadia A, Kellermayer MS (2004) Differential actin binding along the PEVK domain of skeletal muscle titin. J Cell Sci 117:5781-5789

70. Neagoe C, Kulke M, del Monte F, Gwathmey JK, de Tombe PP, Hajjar RJ, Linke WA (2002) Titin isoform switch in ischemic human heart disease. Circulation 106:1333-1341

71. Neagoe C, Opitz CA, Makarenko I, Linke WA (2003) Gigantic variety: expression patterns of titin isoforms in striated muscles and consequences for myofibrillar passive stiffness. J Muscle Res Cell Motil 24:175-189

72. Obermann WM, Gautel M, Weber K, Furst DO (1997) Molecular structure of the sarcomeric $\mathrm{M}$ band: mapping of titin and myosin binding domains in myomesin and the identification of a potential regulatory phosphorylation site in myomesin. EMBO J 16:211-220

73. Opitz CA, Kulke M, Leake MC, Neagoe C, Hinssen H, Hajjar RJ, Linke WA (2003) Damped elastic recoil of the titin spring in myofibrils of human myocardium. Proc Natl Acad Sci USA 100:12688-12693

74. Opitz CA, Leake MC, Makarenko I, Benes V, Linke WA (2004) Developmentally regulated switching of titin size alters myofibrillar stiffness in the perinatal heart. Circ Res 94:967-975 
75. Peng J, Raddatz K, Molkentin JD, Wu Y, Labeit S, Granzier H, Gotthardt M (2007) Cardiac hypertrophy and reduced contractility in hearts deficient in the titin kinase region. Circulation 115:743751

76. Pizon V, Iakovenko A, Van Der Ven PF, Kelly R, Fatu C, Fürst DO, Karsenti E, Gautel M (2002) Transient association of titin and myosin with microtubules in nascent myofibrils directed by the MURF2 RING-finger protein. J Cell Sci 115:4469-4482

77. Preetha N, Yiming W, Helmes M, Norio F, Siegfried L, Granzier H (2005) Restoring force development by titin/connectin and assessment of Ig domain unfolding. J Muscle Res Cell Motil 26:307-317

78. Puchner EM, Alexandrovich A, Kho AL, Hensen U, Schäfer LV, Brandmeier B, Gräter F, Grubmüller H, Gaub HE, Gautel M (2008) Mechanoenzymatics of titin kinase. Proc Natl Acad Sci USA 105:13385-13390

79. Puchner EM, Gaub HE (2010) Exploring the conformation-regulated function of titin kinase by mechanical pump and probe experiments with single molecules. Angew Chem Int Ed Engl 49:1147-1150

80. Pyle WG, Solaro RJ (2004) At the crossroads of myocardial signaling: the role of Z-discs in intracellular signaling and cardiac function. Circ Res 94:296-305

81. Radke MH, Peng J, Wu Y, McNabb M, Nelson OL, Granzier H, Gotthardt M (2007) Targeted deletion of titin N2B-region leads to diastolic dysfunction and cardiac atrophy. Proc Natl Acad Sci USA 104:3444-3449

82. Rief M, Oesterhelt F, Heymann B, Gaub HE (1997) Single molecule force spectroscopy on polysaccharides by atomic force microscopy. Science 275:1295-1297

83. Sebestyén MG, Wolff JA, Greaser ML (1995) Characterization of a $5.4 \mathrm{~kb}$ cDNA fragment from the Z-line region of rabbit cardiac titin reveals phosphorylation sites for proline-directed kinases. J Cell Sci 108:3029-3037

84. Shabarchin AA, Tsaturyan AK (2010) Proposed role of the Mband in sarcomere mechanics and mechano-sensing: a model study. Biomech Model Mechanobiol 9:163-175

85. Sheikh F, Raskin A, Chu PH, Lange S, Domenighetti AA, Zheng M, Liang X, Zhang T, Yajima T, Gu Y, Dalton ND, Mahata SK, Dorn GW 2nd, Heller-Brown J, Peterson KL, Omens JH, McCulloch AD, Chen J (2008) An FHL1-containing complex within the cardiomyocyte sarcomere mediates hypertrophic biomechanical stress responses in mice. J Clin Invest 118:3870-3880

86. Sorimachi H, Freiburg A, Kolmerer B, Ishiura S, Stier G, Gregorio CC, Labeit D, Linke WA, Suzuki K, Labeit S (1997) Tissue-specific expression and alpha-actinin binding properties of the Z-disc titin: implications for the nature of vertebrate Z-discs. J Mol Biol 270:688-695

87. Terui T, Sodnomtseren M, Matsuba D, Udaka J, Ishiwata S, Ohtsuki I, Kurihara S, Fukuda N (2008) Troponin and titin coordinately regulate length-dependent activation in skinned porcine ventricular muscle. J Gen Physiol 131:275-283

88. Trombitas K, Granzier H (1997) Actin removal from cardiac myocytes shows that near $\mathrm{Z}$ line titin attaches to actin while under tension. Am J Physiol 273:C662-C670

89. Trombitas K, Greaser M, Labeit S, Jin JP, Kellermayer M, Helmes M, Granzier H (1998) Titin extensibility in situ: entropic elasticity of permanently folded and permanently unfolded molecular segments. J Cell Biol 140:853-859

90. Tskhovrebova L, Trinick J (2010) Roles of titin in the structure and elasticity of the sarcomere. J Biomed Biotechnol 2010:612482

91. Tskhovrebova L, Trinick J, Sleep JA, Simmons RM (1997) Elasticity and unfolding of single molecules of the giant muscle protein titin. Nature 387:308-312

92. Warren CM, Krzesinski PR, Campbell KS, Moss RL, Greaser ML (2004) Titin isoform changes in rat myocardium during development. Mech Dev 121:1301-1312

93. Willis MS, Schisler JC, Portbury AL, Patterson C (2009) Build it up-tear it down: protein quality control in the cardiac sarcomere. Cardiovasc Res 81:439-448

94. Witt CC, Burkart C, Labeit D, McNabb M, Wu Y, Granzier H, Labeit S (2006) Nebulin regulates thin filament length, contractility, and Z-disk structure in vivo. EMBO J 25:3843-3855

95. Witt SH, Granzier H, Witt CC, Labeit S (2005) MURF-1 and MURF-2 target a specific subset of myofibrillar proteins redundantly: towards understanding MURF-dependent muscle ubiquitination. J Mol Biol 350:713-722

96. Yamasaki R, Berri $\mathrm{M}$, Wu Y, Trombitas K, McNabb M, Kellermayer MS, Witt C, Labeit D, Labeit S, Greaser M, Granzier $H$ (2001) Titin-actin interaction in mouse myocardium: passive tension modulation and its regulation by calcium/S100A1. Biophys J 81:2297-2313

97. Young P, Ferguson C, Banuelos S, Gautel M (1998) Molecular structure of the sarcomeric Z-disk: two types of titin interactions lead to an asymmetrical sorting of alpha-actinin. EMBO J $17: 1614-1624$

98. Zou P, Pinotsis N, Lange S, Song YH, Popov A, Mavridis I, Mayans OM, Gautel M, Wilmanns M (2006) Palindromic assembly of the giant muscle protein titin in the sarcomeric Zdisk. Nature 439:229-233 